\title{
War against antimicrobial resistance
}

\begin{abstract}
Resistance to antimicrobials is a global threat that demands immediate response. The excessive use and misuse of antimicrobials has led to the development of multidrug resistant strains of microorganisms. As the consumption rate of these drugs increases, the resistance rate also increases, resulting in high cost of medical treatment and increased mortality rate. To war against antimicrobial resistance, the biochemical and genetic mechanism of resistance in microorganisms is a key factor to be considered. Recent articles from reputable journals/archives including PubMed, Scopus, Google Scholar, ResearchGate etc were retrieved and studied and we discovered that much have been researched on the importance of drugs in the treatment of antimicrobial resistance and in recent decade researchers have been delving into important botanicals that could address multidrug resistance with several opinions. Researchers have opinionated that phytoconstituents could be potential drugs that would address antimicrobial resistance with little or no side effect and they are relatively cheap and readily available to low-income people. However, the future is largely going to be machine driven and little have been reported on the emerging technologies that could adequately tackle this menace of antimicrobial resistance. Here we succinctly discussed how some of these promising technologies could be employed in combating the resistance of microorganism to antibiotics; the use of vaccine technologies, the use of Artificial Intelligence coupled with machine learning algorithms, the use of nanosystems and the use of CRISPR-cas technology.
\end{abstract}

Keywords: antimicrobial resistance, multidrug resistance (MDR), vaccine technologies, artificial intelligence, CRISPR-cas
Volume 8 Issue 4 - 2020

\section{Sunday Temitope Akinwotu, Oluwaseun Fapohunda}

Department of Biochemistry, Adekunle Ajasin University, Nigeria

Correspondence: Oluwaseun Fapohunda, Department of Biochemistry, Adekunle Ajasin University, Akungba Akoko, PMB 00I, Nigeria, Tel +2347062998896, Email oluwaseun. fapohunda@aaua.edu.ng, fapohundaoluwaseun97@gmail.com

Received: August 03, 2020 | Published: August 3I, 2020

\section{Introduction}

Antimicrobial resistance (AMR) refers to the capacity of a microbe to inhibit the action of a drug/medication that has once efficiently treated a microbe. ${ }^{1}$ The phrase antibiotic resistance is a subclass of AMR which involves the resistance of bacteria to antibiotics. There is difficulty in treating resistant microbes because they require increase in doses of antimicrobials or other options of medications. The resistance of microorganisms (e.g. bacteria, viruses, fungi and parasites) occurs when there are changes as a result of exposure to antimicrobial drugs like antifungals, antibiotics, antivirals, anthelminthics, antimalarials etc. Microorganisms are sometimes referred to as "superbugs" because of their ability to give rise to antimicrobial resistance., ${ }^{2,3}$ Rocha and co-workers ${ }^{4}$ reported the public health concern in the rising factor of antimicrobial resistance and one of the vital warfare to combat this resistance is Antimicrobial Stewardship, but explicit action on its effects is not strongly evident. According to the report made by CDC, every year in the United States, the rate of antibiotic resistant infections is over 2.8 million which results to the mortality of over 35,000 individuals. ${ }^{2}$ Rapid increased number of multidrugresistant (MDR) microbes threatens the modern antimicrobial therapy, with the defect in the technical innovation which is an obstacle in the advancement of new drugs to combat resistance rising rate. ${ }^{5}$ Medicine, plants and production of animals have been completely revolutionized by antibiotics. Furthermore, the prevention of infection and its control, the use of antimicrobials with gaps, incorrect use, excessive use, absence of standard medicine with quality and non-sufficiency in investment have caused gamble in the progress of treating infectious diseases. ${ }^{6}$ As the rate of antibiotics consumption increases, there is also an elevated resistance to treatment in microorganisms. Modern healthcare which is solely dependent on efficient antibiotics coupled with medical operations of great standard to hinder and cure infections is weakened by Antibiotic resistance. ${ }^{7}$ The knowledge of biochemistry and genetics of resistance is significant in composing plans and developing technologies that will shorten how resistance spreads, and its sudden uprising. It will also help to design a forwardlooking curative solutions in opposition to MDR organisms. ${ }^{8}$

\section{Putative mechanism of antimicrobial resistance}

Understanding the biochemical and genetic basis of how microbes resist antimicrobials will help a long way to fight the war against Antimicrobial Resistance. Microorganisms such as bacteria, virus, fungi and parasite exhibit different mechanisms to cause resistance to antimicrobials. Some of these mechanisms are discussed below:

\section{In bacteria}

Antibiotics undergo resistance when in contact with bacteria and this is accomplished in four major ways: (1) limiting drug uptake; (2) drug target modification; (3) drug inactivation; (4) drug efflux pumps. The mechanism of resistance used by gram negative bacteria varies from that of gram positive bacteria. All the four mechanisms of resistance are used by gram negative bacteria while gram positive bacteria uncommonly use limiting drug uptake (because of the absence of lipopolysaccharide on the outer membrane) and do not have the ability to carry out some types of drug efflux pump mechanism. ${ }^{9}$

(1) Limiting drug uptake: The presence of lipopolysaccharide layer in gram negative bacteria hinders the entry of specific types of molecules. This allows the bacteria to develop innate resistance to some specific types of large antimicrobials. ${ }^{10}$ The outer membrane of mycobacteria has high content of lipids which makes hydrophobic drugs (e.g. fluoroquinolones and rifampicin) diffuse easily into the cell but restricts hydrophilic drugs from entering into the cell $\mathrm{s}$ 
(2) Drug target modification: The site of target in bacteria changes as a result of gene mutation. Bacteria modifies drug target by causing alteration in the $30 \mathrm{~S}$ and $50 \mathrm{~S}$ subunits of the ribosome, thus, affecting the synthesis of protein which results into drug resistance i.e. aminoglycosides bind the $30 \mathrm{~S}$ subunit of ribosome while macrolides, lincosamides, streptogramin $\mathrm{B}$ and chloramphenicol binds to 50S subunits of ribosome to inhibit protein synthesis. However, alteration of Penicillins binding protein (PBP)is a common mechanism of resistance for gram positive bacteria while $\beta$-lactamases production is a mechanism of resistance for gram negative bacteria. Mutation in the PBP causes reduction of affinity to $\beta$-lactam antibiotics. ${ }^{11}$

(3) Drug inactivation: Bacteria inactivates antibiotics in two ways either by degradation of the antibiotics or by transferring a chemical group to the antibiotics e.g. acetyl group, phosphoryl group and adenyl group. The three main enzymes that inactivates antibiotics are $\beta$-lactamases (which hydrolyses mostly all $\beta$-lactams that have ester and amide bond e.g. penicillin, cephalosporins etc.), aminoglycoside-modifying enzymes and chloramphenicol acetyltransferases. ${ }^{12}$ Acetylation mechanism is mostly used against chloramphenicol, flouroquinolones, aminoglycosides and streptogramins. Phosphorylation and adenylation are normally used against aminoglycosides. ${ }^{13}$

(4) Drug efflux pumps: Membrane proteins that facilitate the movement of antibiotics across the cell to make balance in their intracellular concentrations are known as efflux pumps. Efflux mechanism also helps to pump out the antimicrobials that are entering the cell before reaching their target. ${ }^{14}$ Efflux pumps have five major families which includes the ATP-binding cassette family (ABC), major facilitator super family (MFS), small multidrug resistant family (SMR), resistance-nodulationcell-division family (RND), multidrug and toxic compound extrusion family (MATE). ${ }^{8}$

\section{In viruses}

Viral infections are treated with some unique antiviral drugs to reduce the load of the virus in the body system (Viral Load). Antiviral drugs hinder viruses from replicating by the inhibition of the cycle of target e.g. some serve as protease inhibitors, reverse transcriptase inhibitors, integrase inhibitor, polymerase inhibitor etc. Viral infection like hepatitis B, Hepatitis C, HIV, influenza etc. are treated with antivirals e.g. lamivudine, nevirapine, cidofovir, efavirenz, ribavirin, duranavir etc. Some of these virus strains have created resistance towards the drugs prescribed..$^{15}$ Antiviral resistance is caused by gene a mutation that codes the drug target protein.HIV antiviral resistance is difficult to overcome; even the strains of multi-drug resistance have developed. HIVResistant strains arise quickly when only one antiviral drug is being used. A form of therapy refers to as combination therapy which involves the use of three or more drugs concurrently have gone a long way in helping the control of this resistance. Moreover, brand new drugs are necessary because strains of HIV drug-resistance are developing continually. ${ }^{16}$

\section{In fungi}

Fungal infections cause elevated unwholeness and death rate in individual with compromised immune system. Aspergillus fumigatus, Candida and Cryptococcus neoformans cause most of the fungal infections with an occurrence of resistance in them all. Treatment of individuals with compromised immune system has resulted into an increased level of Multidrug resistance in fungi due to the extensive use of antifungal drugs. ${ }^{17}$ Antifungals that are majorly prescribed are classified into three types which are the polyenes, echinocandins and azoles. Pores are produced in the cell membrane of fungi when in contact with Amphotericin B and other polyenes resulting to fungi cell death. Inhibitionof $\mathrm{C} 14-\alpha$ sterol demethylaseand disruption the structure of the fungal cell membrane is caused by fluconazole and other azoles. The synthesis of cell wall in fungi is destroyed by inhibiting $\beta-1,3-\mathrm{D}$-glucan synthesis bycaspofungin and other echinocandins. A study in 2017 shows that the mechanism of resistance to fluconazole in $C$. auris is by point mutation and the number of ERG11 gene which codes for $14 \alpha$-lanosterol demethylase in the biosynthetic pathway of ergosterol increases. ${ }^{18}$

\section{In parasite}

Human pathogens like plasmodium, leishmaniasis, trypanosomiasis etc. are protozoan parasites that give rise to diseases. Parasites of malaria have created resistance to anti-malarial drugs e.g. artemisin in which resulted into the continuous development of new drugs with high efforts. ${ }^{19}$ Protozoan parasites undergo adaptation and resistance to many standard drugs. These adaptations include reduction in drug uptake (diminazene aceturate in T. brucei), metabolic bypass up-regulation (e.g. methotrexate in Leishmania), failure in prodrug activation (e.g. nifurtimox in T. cruzi), increase in drug efflux (e.g. chloroquine in malaria), failure in target production and target enzyme mutation (e.g. antifolates in malaria). Drug resistance for some drugs happens faster than for others despite the different mechanisms with which protozoa can develop resistance. In some cases, gene mutation or deletion is enough for disrupting the sensitivity of drugs, followed by secondary mutation to develop resistance of higher level. ${ }^{20}$

\section{Public health factors contributing to antimicrobial resistance}

a. Quality of drug and its dispenser: There is absence of suitable regulations in selling antimicrobials which is an important quality in the incorrect use and access to antimicrobials. Many antimicrobials can be bought without physician's prescription and are commonly given out on streets by untrained personnel, this occurs in most developing countries and are being sold by drug vendors just to make a sale. Some pharmacies even work with no license and the public have more access to them because it does not waste time, fee for consultation is free and treatments are negotiable according to the money present with the patient. In the developing countries like in Africa, retail pharmacies serve as a principal level of outpatient care, thereby giving services without authority e.g. in the prescription, diagnosis, consultation and issuing of drugs. ${ }^{21}$

b. Healthcare professionals: The providers of healthcare play an important part in preventing and treating of diseases, but if their practices are not based on evidence it will endanger their profession and the lives of patients. For instance, the practice of prescribing antimicrobial differs amongst physicians of different countries. In few cases, the prescription of antimicrobials are not appropriate i.e. wrong doses or wrong drug which will in turn result in resistance development against such antimicrobials. ${ }^{22}$

c. Patients: The act of compliance is of great significance towards the development of antimicrobial resistance. Some patients intentionally or mistakenly miss the dose of drugs. This act exposes surviving microbe to sub-curative drug concentration, causing elevation in the opportunity of developing resistance..$^{23}$ 
d. Sanitation and hygiene: Unusual environmental state such as inadequate sanitation and overcrowding chip in the spread and transmission of resistant microorganisms. Resistant pathogens are transmitted by contact from person to person, through vectors, contaminated food and water. Resistant microorganisms will be reduced if there is improvement in sanitation and basic hygiene. $^{24}$

e. Fake drugs: Some sellers now peddle fake drugs around and this heinous act has resulted in devastating health effects including drug resistance.

\section{Possible solutions to antimicrobial resistance \\ The use of vaccine technologies}

The availability of technologies today can aid in the development of vaccines that can proffer solutions to antimicrobial resistance. Antimicrobial resistant vaccines are one of the major vaccines that can be developed technically. The comprehensive and the most promising technologies to combat Antimicrobial resistance are described below:

(a) Reverse vaccinology: This refers to the process of sequencing pathogens genome using computer programs to reveal genomeencoded antigens which will be very hard for ordinary technology to expose..$^{25}$ This kind of technology is called reverse vaccinology due to the fact that in the history of vaccine development, it was the first time after a long period of time (i.e. two centuries) that scientists explore new vaccines beginning from the information gotten on a computer in place of microorganism growing. The recent development of newly licensed four-component meningococcus $B$ vaccine (4CMenB) revealed that this technology is efficient in selecting antigens of bacteria which can give broad protection versus bacterial pathogens of different antigens. Essentially, the selection of antigens process begins with genomic information analysis on circulating strains. Antigens of interest are picked on the basis of sequence conservation and forecasted surface exposure which are also distinctly stated as recombinant proteins and are experimented in preclinical models for their strength to trigger functional antibodies. Lastly, more promising antigen of interest are joined together to obtain the best possible result based on the data gotten from molecular epidemiology. Three recombinant antigens i.e. fHbo, NadA and NHBA are used in the case of $4 \mathrm{CMenB}$ by the induction of serum antibodies that are able to kill bacteria versus strains which expresses a conserved form of each corresponding protein joined with the OMV from New Zealand meningococcus $B$ Strain, the antibodies induced has the ability to kill the strains showing a conserved form of the outer membrane protein PorA. Parikh and co-workers ${ }^{26}$ reported that $4 \mathrm{CMenB}$ has been introduced recently to all the infants in UK, it demonstrates effectiveness of $83.9 \%$ against all strains of MenB. This achievement of $4 \mathrm{CMenB}$ shows that the section of antigen process can be applied to all bacteria pathogens that shows antimicrobial resistance. The only condition for reverse vaccinology is the availability of the organism's whole genome sequence. In regards to the onset of reverse vaccinology, the genome sequences of anenormous number of isolates may be analyzed for homology. The whole genome sequence is necessary for the forecast of surface proteins and epitopes; which is the significant element of reverse vaccinology for the development of an effective vaccine. This implies that reverse vaccinology is beneficial for the development of epitope vaccines to combat pathogen resistance. ${ }^{27}$ Maryam and co-workers carried out an insilico study on novel Coronavirus disease (COVID-19) with its recent outbreak in Wuhan in December 2019 using a reverse vaccinology approach to design an up to standard candidate vaccine against COVID-19, by exploiting the programs of reverse vaccinology. The result shows that the candidate vaccine can stimulate both the cellular and anitibody-mediated immunity, considering the fact that B-cell and T-cell epitopes have been chosen in the final model. ${ }^{28}$

(b) Structural vaccinology: Since it is possible to carry out isolation of monoclonal antibodies ( $\mathrm{mAbs}$ ) and being able to figure out the structure of antigen-antibody complexes, reveal a new promising area in which information of structure can be used in enhanced antigen engineering. ${ }^{29}$ McLellan and co-workers ${ }^{30}$ reported that this technology in its early stage has given birth to vast changed antigen for respiratory syncytial virus (RSV) by making stable the RSV's F protein in perfusion conformation. This task was not possible when ordinary technology was being used. In the improvement of HIV outlook, similar process is being used and also to produce general vaccines for influenza. Human mAbs have been crucial step to reveal novel protective antigens e.g. CMV pentameric complex, ${ }^{31}$ and the RSVF protein perfusion conformation. ${ }^{30}$ Despite the target of most antigen application design is viral pathogens, the technology is also applicable to bacteria, giving room for novel bacteria antigens to be identified with enhanced security within strains of bacteria. However, developments in the engineering of protein now give way to show recombinant antigens in multimeric structures of great order making use of different protein scaffolds. ${ }^{32}$ The display of antigens on nanoparticles can be logically developed to enhance protein density and formation of accurate multimers in right conformation. Nidhi and co-workers ${ }^{33}$ conducted a study that designed a next-generation novel multi epitope subunit vaccine versus deadly disease, anthrax by researching five virulent and secretory proteins. Also, the potential epitopes which was included is available in all immunogenic proteins conveyed for anthrax and speculated to create a potential chimeric vaccine.

(c) Phage therapy: Viruses which infect bacteria are called phages and they are the most notable life form on earth. Before the discovery of penicillin, phages were being used as antibiotics. There are various kinds of phages virus, but the most occurring process for Bacteria therapy include lytic phages; the phages that give rise to breakdown of cell and eventually causes the death of the bacterial cell. After the binding of Bacteria to phages, they inject either the RNA or DNA which now be used as host cofactor and host enzymes for replication. Lytic phages in holds the enzyme called lysin whose function is to carry out digestion of bacteria proteins resulting to extreme damage. The use of phages for treatment is significant because, an enormous quantity of phages can be administered in minute dose and the bacteria causing the infection will undergo replication to give rise to more phages in situ. However, phages have high specificity for bacteria, which implies that there is no strong effect on the cells of mammals but unluckily, it (specificity) limits the phages. The target of one phage is limited to number of bacteria strain which implies that many various phages are required to treat one species of bacteria. Investigations are being done by researchers to expand the specificity of phages, ${ }^{34}$ which can result into reduction in the number of phages required per species and modify this process. Another limitation is how to bring these phages to their action site since they do not have suitable protein kinase properties. Rastogi and coworkers ${ }^{35}$ 
reported various studies to treat different bacterial infections with phages delivered by parental transdermal, oral, topical, intranasal and auditory routes. Producing curative phages involves the knowledge of each gene (and protein) function. Still, large numbers of phage genomes are not even totally sequenced let alone the understanding of molecular functions of their gene products. This is the time for molecular biologists and biochemists to be in one accord with other microbiologists and clinicians in this non-ending battle against pathogens. Governments need to be aware that the basic knowledge of the approach involved in AMR and phage-therapy are as significant, maybe even more significant than researching intermediate solutions. ${ }^{36}$

(d) Antibody therapy: Considering the fact that antibodies can target bacteria, it is not a surprise that important measures had been put in place towards the development of antibody therapy to replace antibiotics. Antibody therapy has successful application in other therapeutic areas e.g. inflammatory diseases. Also, widely neutralizing monoclonal antibodies (mAbs) are having great influence on HIV research recently ${ }^{37}$ In previous years, the utility of monoclonal antibodies has been suggested as an option in treating MDR pathogens, because of their targeted specificity versus the bacterial pathogen, their finite possibility of developing resistance and their capacity to behave cooperatively with antibiotics. ${ }^{38} \mathrm{~A}$ limitation to treatment of bacterial infections by antibodies is that antibodies have finite spectrum and its target is a little number of strains of definite species, on a contrary, antibodies designed as antibiotics must have the ability to target an array of bacteria. Researchers can now modify antibodies with the new technology which increases the power of a one antibody to bind two targets (bispecific $\mathrm{mAb}$ ) and also decreases the total number of mAbs required for treatment of infection. Medimmune illustrated this process through the enhancement of bispecific $\mathrm{mAb}$ to target a microorganism ( $P$. aeruginosa) whose targets are Psl and PcrV. ${ }^{39}$ Another process is targeting virulence toxins or proteins which are being produced by the bacteria to the extent that it is no longer harmful to the host cell. ${ }^{40}$ The limitations to this process are that they are only conducted on clinical trials whose target is to treat a specific pathogen, and the expense of mAbs goods. Using mAbs to treat hospital acquired infections e.g. K. pneumonia, P. aeruginosa or Acinetobacter in patients with high risk can be a more practical process compared to the vaccination for the pathogens where infection can be proportionally scarce in most people lives till they move into an environment with high risk e.g. the ICU (Intensive Care Unit). ${ }^{41}$ With the advancement in diagnostic technologies and antibody engineering with manufacturing process that lowers goods cost, in the future this can be an area of importance. Out of all the processes explained, mAbs presumably have the best chance of treating AMR efficiently, though the treatment of each $\mathrm{mAb}$ will be of limit to a specific bacteria species. Advancements in antibody therapy, affixed with clinical requirements, may absolutely motivate more research with funding to the $\mathrm{mAb}$ approach. As a matter of fact, it may become a speedy pathway than small-molecule advancement and per adventure radically change infectious medicine as was proposed years ago. ${ }^{42}$

\section{The use of artificial intelligence application with machine-learning algorithm}

Artificial Intelligence (AI) involves the use computer programs to reproduce human level of intelligence, knowledge and conscience.
This has a great success in science and also for the development of new drugs. Massachusetts Institute of Technology (MIT) researcher makes use of pioneering machine learning algorithm to discover new kinds of antibiotics from 100 of millions molecules in the year 2019. Within this approach, the AI programme discovered one (Halacin), the first antibiotic discovered, which target a broad array of bacteria, involving tuberculosis and strains regarded as impossible to treat. Though, AI has been used before in the discovery process of antibiotics, but this was the first time new types of antibiotics has been discovered from the scratch outside former assumptions by human. The use of computer model for in silico examination is not a new approach, but until now, the accuracy of this model was not sufficient to change the discovery of drug. It deserves to say that small number of antibiotics has been created in the last decade and many of these antibiotics exhibits little difference from the ones that exists. Computer models in the past were not accurate enough to change greatly the discovery of drugs. Beforehand, chemical molecules were portrayed as vector showing that a certain group of chemical molecules is present or not, but the recent neutral networks modify the situation drastically. Neutral networks are algorithms designed like the human brain to identify patterns. They explain sensory data by a form of machine cognition, grouping and labelling raw input data. They can also study these illustrations in an automatic manner and mapping the molecules into vector which are afterwards used to forecast their attributes. ${ }^{43}$ Lui and co-workers ${ }^{44}$ carried out a research by applying the Support Vector Machine (SVM) and Set Covering Machine (SCM) algorithm to precisely foretell their phenotypic appear ance versus five agents of antimicrobial which are: Tetracycline, Ampicillin, Sulfisoxazole, Trimethoprim, and Enrofloxacinfrom the whole genomes of 96 isolates of A. pleuropneumoniae. Amidst the five agents of antimicrobial, the resistant activity of $A$. pleuropneumoniae versus tetracycline is more difficult than the others. 58 phenotype resistant strains were available, with 50 isolates transporting tet (B), 5 isolates transporting tet $(\mathrm{H})$, and in 3 isolates, the resistance genes of tetracycline were not noticed. ${ }^{44}$

\section{The use of nanosystems}

Nanomedicine (medicine of nanoparticles) plays a significant role in heightening ability of therapeutics in existence by developing the physicochemical properties and antibiotics stability, putting forth an opportunity of biofilm internalization, delaying the distribution of antibiotics, as well as the ability of the delivery Target to the site of infection and consequently enhancing the circulation within the system with a decline of side effects in respect to the related free drugs. ${ }^{45}$ The main factors that influences the important process of nanosystems such as bio-distribution, intracellular uptake or clearance are the physicochemical properties, surface charge, particle size and solubility. Nanoparticles allows good drug development ability of both lipophilic (lipid soluble) and hydrophilic (water soluble) antibiotics and thus improve antibacterial effects. ${ }^{46}$ As well as more anticipated, cellular internalization of the antibiotics loaded nanosystems was accomplished by passing of the reticuloendothelial system. ${ }^{47}$ The charge on the surface of nanosystems and the zeta $(\zeta)$-potential motivates proteins interactions, interactions with various parts of tissues which in turn affect its bio-distribution within the cells and uptake. Host cells e.g. macrophages with negatively charged nature have affinity for positively charged nanosystems in comparison with the negatively charge and ones with no charge. ${ }^{48}$ The properties of nanosystems not to be soluble in water (hydrophobicity) show an important function in drug delivery targeting which is in relation to the interactions with phospholipid layer of the bacterial cell membrane. ${ }^{45}$ On the other hand, nanosystems that have the 
ability to dissolve in water (hydrophilic) attract less with opsonins and in turn making the circulation of blood long in comparison with hydrophobic nanosystems. ${ }^{49}$ As a result, the improved activity of nanosystems in the system delivery of antibacterial drugemerges from different mechanisms which includes their capacity to enhance the physicochemical properties of the entrapped antibacterial drugs, their favoured retention around the cytoplasm, their electrostatic affection towards the bacterial cell membrane, elevated oxidizing ability and production of ROS (reacting oxygen species), the prevention of unwelcomed interactions, coverage of antibacterial drugs to avoid degradation and the well use of antibacterial drugs through patient more acceptable pathway. ${ }^{50}$ It is quite interesting that nanoparticles do not only enhance antibacterial agents activity but also curb the development of resistance by having breakthrough over the resistance strategy developed by bacteria which involves the decomposition of drug by $\beta$-lactaoamase, making bacterial cell wall thick etc ${ }^{51}$ Reports have shown that antimicrobial peptides (AMPs) can also serve as delivery medium for compounds of great biological effects. ${ }^{52}$ The making of hybrid peptides in which an AMP is capable of passing through membranes is joined with another AMP which has the capacity of acting within the cell after been transferred to the membrane. This can be a breakthrough in the enhancement of antibacterial activity of present AMPs..$^{53}$ More complicated peptidic systems are referred to as antimicrobial peptide dendrimers (AMPDs), based on branched polymers consisting of several peptides covalently joined to a core. These polyvalentimitative peptide have acquired mental focus in the previous years over because of their elevated antibacterial activity, increased resistance to degradation, likely because of significant steric hindrance, with the increased concentration of bioactive units per dendrimer molecule. ${ }^{54}$ In times ahead, the merger of AMPDs with any other nanoparticles may make even more efficient and inspiring combat in against multidrug resistant infections. ${ }^{53}$

\section{The use of CRISPR-Cas technology system}

The term CRISPRs (clustered regularly interspaced short palindromic repeats) refers to the adaptive immune systems that are originated from bacteria and archaea. This system (CRISPR-Cas) make use of RNA to recognize its target DNA and the Cas enzyme to gradually destroy the nucleic acids, so their requirement is only one protein for binding purpose and cleavage purpose. ${ }^{55}$ CRISPRcas system degrades foreign genetic elements in three steps. The first stage is Adaptation or spacer acquisition in which the spacer sequence after recognition is fused into the array of CRISPR. The second stage is biogenesis or expression of CRISPR RNA (crRNA) which involves the transcription of pre-CRISPR RNA (pre-crRNA) catalyzed by RNA polymerase. The pre-crRNA is then cleaved into few numbers of crRNA which are also known as guide RNAs by specific enzymes referred to as endoribo nucleases. The last step is the Interference in which crRNAs recognize and form base pair specific to foreign RNA or DNA and are complementary to each other resulting to the cleavage of the foreign crRNA nucleic acid complex. However, if mutation occurs in the proto-spacer adjacent motif (PAM) or there is mismatch in between the spacer and invader's DNA, it will result in the termination of the cleavage and the host is vulnerable to infection. ${ }^{56}$ The mechanism of CRISPR-Cas system, its region and the Cas genes contents are grouped into three (3) main types (I, II and III) and eleven(11) subtypes (I-A to I-F, II-A to IIC, and III-A to III-B). The most studied system is Type II system and its mechanism is the most illustrated system among the three systems. ${ }^{57}$ Various studies have proved the involvement of CRISPRcas system in antimicrobial resistance e.g. this system enhanced the promotion of envelope integrity of $F$. novicida by the regulation of Bacteria Lipoprotein leading to the growth of resistance in contrast to several membrane stressors, involving antibiotics. ${ }^{58}$ Another study reveals the relationship between competence systems (which promotes acquisition of genes) and CRISPR system. For example, Aggregatibacter actinomycetemcomitans competent strains possess CRISPR-cas systems while CRISPR immune system was lost in non-competent bacterial strains. ${ }^{59}$ This shows that the development of competence system and CRISPRs enhances the rise of genes of different species in bacteria. ${ }^{56}$ Two studies illustrated that CRISPRCas 9 gene can be transported utilizing plasmids packaged in phage capsids referred to as phagemids to selectively terminate the bacterial pathogens; E. coli and Staphylococcus aureus.$^{60}$ However, there are challenges faced with the use of CRISPR-Cas system to war against AMR, but there are solutions to tackle some of these challenges. The most persistent challenge facing this system is discovering suitable delivery vector that is engineered for its distinct purpose. For instance, phage-mediated delivery can possibly been option in acute infection. Although, strains of the same bacterial species frequently change in phage susceptibility, and different delivery vector will be suitable. ${ }^{61}$ Kiga and co-workers ${ }^{62}$ used the motley RNA cleavage capability of CRISPR-Cas 13a by means of recognizing target RNA by CRISPRRNA (crRNA), which leads to death in the host cell to spawn a new form of sequence-specific bacterial antimicrobials. For the delivery of CRISPR-Cas13a to its bacteria of target, CRISPRCas13a was packaged into the carrier phage capsid utilizing phage-inducible chromosomal island (PICI) packaging system for E. coli, and $S$. aureus Pathogenicity Island (SaPI) packaging system for $S$. aureus. Because synthesized CapsidCas13a does not transport phage genome, but is a part of the categories of nucleic acids drug or gene drug, which will be out into use as a therapeutic drug.

\section{Conclusion}

Though, there are still some effective antimicrobials, but the rate of developing resistance to them is high, thus, the use of technologies to war against antimicrobial resistance is inescapable. Using vaccine technologies (reverse vaccinology, structural vaccinology, phage therapy and antibody therapy) will bring succor in developing vaccines technically to effectively treat AMR. The only technique required in reverse vaccinology is the organism whole genome sequence which can be screened for homology. The whole genome sequence is needed for epitopes and other proteins prediction which is a significant requirement for candidate vaccine development. Also, combining phage therapeutics with antibiotics shows a promising approach for drug development in combating antimicrobial resistance. Artificial Intelligence with machine learning algorithm had shown great potential in targeting wide array of bacteria which will improve the development of antimicrobials with no strains of resistance. Also, the use of nanoparticles as antimicrobials to target the delivery site will be of great significance to combat resistance. CRISPR-cas technology has a striking enthusiasm in circumventing the challenges faced with antimicrobial resistance. These approaches have illustrated how resistant microorganisms can be tackled to enhance the advancement of antimicrobials.

\section{Acknowledgments}

None.

\section{Conflicts of interest}

Authors declare that there is no conflict of interest. 


\section{References}

1. About Antimicrobial Resistance-Antibiotic/Antimicrobial Resistance. CDC; 2017.

2. Antibiotic Resistance Threats in the United States, 2019. Atlanta, GA: U.S. Department of Health and Human Services, CDC; 2019.

3. WHO. Antimicrobial Resistance; 2018.

4. Rocha-Pereira N, Figueiredo Dias P, Correia S, et al. Persuasive antimicrobial stewardship intervention in the context of a KPC outbreak: a controlled interrupted time series analysis. Antimicrob Resist Infect Control. 2020;9:55.

5. Zalevskaya, O, Gur'eva, Y, Kutchin, A, et al. Antimicrobial and Antifungal Activities of Terpene-Derived Palladium Complexes. Antibiotics. 2020;9(5):277.

6. Lomazzi M, Moore M, Johnson A. et al. Antimicrobial resistance moving forward?. BMC Public Health 2019;19:858.

7. Roope SJ, Richard DS, Pouwels KB, et al. The challenge of antimicrobial resistance: What economics can contribute. Science. 2019;364(6435):eaau4679.

8. Munita JM, Arias CA. Mechanisms of Antibiotic Resistance. Microbiol Spectr. 2016;4(2):10.1128 microbiolspec.VMBF-0016-2015.

9. Wanda CR. An Overview of the antimicrobial resistance mechanisms of bacteria. AIMS Microbiology. 2018;4(3):482-501.

10. Mahon CR, Lehman DC, Manuselis G. Antimicrobial agent mechanisms of action and resistance. In: Textbook of Diagnostic Microbiology, St. Louis: Saunders; 2014. 254-273 p.

11. Kapoor G, Saigal S, Elongavan A. Action and resistance mechanisms of antibiotics: A guide for clinicians. J Anaesthesiol Clin Pharmacol. 2017;33(3):300-305

12. Kumar A, Schweizer HP. Bacterial resistance to antibiotics: active efflux and reduced uptake. Adv Drug Deliver Rev. 2005;57(10):1486-1513.

13. Blair JM, Webber MA, Baylay AJ, et al. Molecular mechanisms of antibiotic resistance. Nat Rev Microbiol. 2015;13(1):42-51.

14. Džidic S, Šuškovic J, Kos B. Antibiotic resistance mechanisms in bacteria: Biochemical and genetic aspects. Food Technol Biotechnol. 2008;46(1):11-21.

15. Lou Z, Sun Y, Rao Z. Current progress in antiviral strategies. Trends in Pharmacological Sciences. 2014;35(2):86-102.

16. Ebrahim O, Mazanderani AH. Recent developments in hiv treatment and their dissemination in poor countries. Infectious Disease Reports. 2013;5(Suppl 1):e2.

17. Costa C, Dias PJ, Sá-Correia I, et al. MFS multidrug transporters in pathogenic fungi: do they have real clinical impact?. Frontiers in Physiology. 2014;5:197.

18. Berkow EL, Lockhart SR. Fluconazole resistance in Candida species: A current perspective. Infect Drug Resist. 2017;10:237-245.

19. Chia WN, Goh YS, Rénia L. Novel approaches to identify protective malaria vaccine candidates. Frontiers in Microbiology. 2014;5:586.

20. De Koning, Harry. Drug resistance in protozoan parasites. Emerging Topics in Life Sciences. 2017;1(6):627-632.

21. Okeke IN, Aboderin OA, Byarugaba DK, et al. Growing problem of multidrug-resistant enteric pathogens in Africa. Emerg Infect Dis. 2007;13(11):1640-1646.

22. Usluer G, Ozgunes I, Leblebicioglu H. Turkish antibiotic utilization study group. A multicenter point-prevalence study: antimicrobial prescription frequencies in hospitalized patients in Turkey. Ann Clin Microbiol Antimicrob. 2005;4:16.
23. Calva J, Bojalil R. Antibiotic use in a periurban community in Mexico: a household and drugstore survey. Soc Sci Med. 1996;42(8):1121-1128.

24. Smith MA, Garbharran H, Edwards MJ, et al. Health promotion and disease prevention through sanitation education in south African Zulu and Xhosa women. J Transcult Nurs Off J Transcult Nurs Soc. 2004;15(1):6268

25. Delany I, Rappuoli R, Seib KL. Vaccines, reverse vaccinology, and bacterial pathogenesis. Cold Spring Harb Perspect Med. 2013;3(5):a012476.

26. Parikh SR, Andrews NJ, Kazim B, et al. Effectiveness and impact of a reduced infant schedule of $4 \mathrm{CMenB}$ vaccine against group $\mathrm{B}$ meningococcal disease in England: A national observational cohort study. Lancet. 2016;388(10061):2775-2782.

27. Kanampalliwar AM, Rajkumar S, Girdhar A, et al. Reverse Vaccinology: Basics and Applications. J Vaccines Vaccin. 2013;4: 194.

28. Maryam E, Mehdi H, Sobhan F, et al. Reverse vaccinology approach to design a novel multi-epitope vaccine candidate against COVID-19: an in-silico study. Journal of Biomolecular Structure and Dynamics. 2020:1-16.

29. Rappuoli R, Bottomley MJ, D’Oro U, et al. Reverse vaccinology 2.0 Human immunology instructs vaccine antigen design. J Exp Med. 2016;213(4):469-481

30. McLellan JS, Chen M, Gordon MJ, et al. Structure-based design of a fusion glycoprotein vaccine for respiratory syncytial virus. Science. 2013;342(6158):592-598

31. Macagno A, Nadia LB, Fabrizia V, et al. Isolation of human monoclonal antibodies that potently neutralize human cytomegalovirus infection by targeting different epitopes on the $\mathrm{gH} / \mathrm{gL} / \mathrm{UL} 128-131 \mathrm{~A}$ complex. J Virol. 2010;84(2):1005-1013

32. Kanekiyo M, Wei J, Hadi M, et al. Self-assembling influenza nanoparticle vaccines elicit broadly neutralizing H1N1 antibodies. Nature. 2013;499(7456):102-106.

33. Nidhi G, Nazia K, Amit M, et al. Structural vaccinology approach to investigate the virulent and secretory proteins of Bacillus anthracis for devising anthrax next-generation vaccine. Journal of Biomolecular Structure and Dynamics. 2019:1-11.

34. Fairhead H, Wilkinson A, Severi E. US Patent WO 2017/174809, A1; 2017.

35. Rastogi V, Verma N, Mishra K, et al. An overview on bacteriophages: A natural nanostructured antibacterial agent. Curr Drug Deliv. 2018;15(1):3-20

36. Loh B, Leptihn S. A Call For a Multidisciplinary Future of Phage Therapy to Combat Multi-drug Resistant Bacterial Infections. Infectious Microbes \& Diseases: 2020;2(1):1-2.

37. Pegu A, Hessell AJ, Mascola JR, et al. Use of broadly neutralizing antibodies for HIV-1 prevention. Immunol Rev. 2017;275(1):296-312.

38. Domenech M, Sempere J, de Miguel, S et al. Combination of Antibodies and Antibiotics as a Promising Strategy Against Multidrug-Resistant Pathogens of the Respiratory Tract. Front Immunol. 2018;9:2700.

39. Di Giandomenico A, Keller AE, Gao C, et al. A multifunctional bispecific antibody protects against Pseudomonas aeruginosa. Sci Transl Med. 2014;6(262):262ra155.

40. Kummerfeldt CE. Raxibacumab: Potential role in the treatment of inhalational anthrax. Infect Drug Resist 2014;7:101-109.

41. Bagnoli F, Payne DJ. Reaction: Alternative modalities to address antibiotic-resistant pathogens. Chem. 2017;3(3):369-372

42. Zurawski DV, McLendon MK. Monoclonal Antibodies as an Antibacterial Approach Against Bacterial Pathogens. Antibiotics. 2020;9(4):155. 
43. Valavanidis A. Artificial Intelligence Application with Machine- learning Algorithm Identified a Powerful Broad-Spectrum Antibiotic. Scientific Reviews. 2020;1:1-24.

44. Liu Z, Deng D, Lu H, et al. Evaluation of Machine Learning Models for Predicting Antimicrobial Resistance of Actinobacillus pleuropneumoniae From Whole Genome Sequences. Front Microbiol. 2020;11:48.

45. Patra JK, Das G, Fraceto L, et al. Nano based drug delivery systems: Recent developments and future prospects. J Nanobiotechnol. 2018;16(1):71.

46. Lombardo D, Kiselev MA, Caccamo MT. Smart nanoparticles for drug delivery application: Development of versatile nanocarrier platforms in biotechnology and nanomedicine. J Nanomater. 2019;2019:3702518.

47. Abed N, Couvreur P. Nanocarriers for antibiotics: A promising solution to treat intracellular bacterial infections. Int $J$ Antimicrob Agents. 2014;43(6):485-496.

48. Dacoba TG, Olivera A, Torres D, et al. Modulating the immune system through nanotechnology. In: Seminars in Immunology. Elsevier: Amsterdam, The Netherlands; 2017.

49. Gustafson HH, Holt-Casper D, Grainger DW, et al. Nanoparticle uptake: The phagocyte problem. Nano Today. 2015;10(4):487-510.

50. Vallet-Regí M, González B, Izquierdo-Barba I. Nanomaterials as Promising Alternative in the Infection Treatment. Int $\mathrm{J} \mathrm{Mol} \mathrm{Sci.}$ 2019;20(15):3806.

51. Baptista PV, McCusker MP, Carvalho A, et al. Nano-strategies to fight multidrug resistant bacteria-“A Battle of the Titans". Front Microbiol. 2018;9:1441.

52. Biswaro LS, da Costa Sousa MG, Rezende TMB, et al. Antimicrobial peptides and nanotechnology, recent advances and challenges. Front Microbiol. 2018;9:855.
53. Martin-Serrano Á, Gómez R, Ortega $\mathrm{P}$, et al. Nanosystems as Vehicles for the Delivery of Antimicrobial Peptides (AMPs). Pharmaceutics. 2019;11(9):448.

54. Manzo G, Serra I, Pira A, et al. The singular behavior of a $\beta$-type semisynthetic two branched polypeptide: Three-dimensional structure and mode of action. Phys Chem Chem Phys, 2016;18(45):30998-31011.

55. Lima R, Del Fiol FS, Balcão VM. Prospects for the Use of New Technologies to Combat Multidrug-Resistant Bacteria. Front Pharmacol. 2019;10:692.

56. Shabbir MAB, Shabbir MZ, Wu Q, et al. CRISPR-cas system: biological function in microbes and its use to treat antimicrobial resistant pathogens. Ann Clin Microbiol Antimicrob. 2019;18(1):21.

57. Ateş A, Tastan C, Ermertcan, S. Alternative Therapies to Antibiotics: CRISPR-Cas antimicrobials. Gene Editing. 2020;1:30-35.

58. Sampson TR, Napier BA, Schroeder MR, et al. A CRISPR-Cas system enhances envelope integrity mediating antibiotic resistance and infammasome evasion. Proc Natl Acad Sci. 2014;111(30): 63-68.

59. Jorth P, Whiteley M. An evolutionary link between natural transformation and crispr adaptive immunity. MBio. 2012;3(5): e00309-e00312.

60. Citorik RJ, Mimee M, Lu TK. Sequence-specific antimicrobials using efficiently delivered RNA-guided nucleases. Nat Biotechnol. 2014;32(11):1141-1145.

61. Pursey E, Sunderhauf D, Gaze WH, et al. CRISPR-Cas antimicrobials: Challenges and future prospects. PLoS Pathog. 2018;14(6):e1006990.

62. Kiga, K, Tan X, Ibarra-Chavez R, et al. Development of CRISPRCas 13a-based antimicrobials capable of sequence-specific killing of target bacteria. Nature Communications. 2020;11:2934. 